\title{
Thermal Fluctuations in Conjugation and their Effect on Calculated Excitation Energies: A Case Study on the Astaxanthin Carotenoid
}

\author{
Jun Wang ${ }^{[a]}$ and Bo Durbeej* ${ }^{*[b]}$
}

A popular approach to the calculation of molecular excitation
energies is to consider only equilibrium geometries and neglect
the effects of thermal motion. Although this static approach is
sensible for molecules with distinct potential-energy minima, its
adequacy relative to dynamical approaches appears not to have
been thoroughly tested. Here, we report a case study investigat-
ing how thermal motion accounted for by molecular dynamics
simulations influences the optically bright state of astaxanthin,
a carotenoid of broad photobiological interest that features

\section{Introduction}

Accurate quantum chemical modeling of electronically excited states of molecular systems is of widespread utility in both basic and applied research. Accordingly, a wealth of insightful benchmark studies has been performed to help identify suitable methods for realizing this goal. Often, such studies focus on the calculation of the energies of excited states, ${ }^{[1-16]}$ although other properties have also been considered, like their geometries, ${ }^{[17-25]}$ vibronic structure ${ }^{[26-30]}$ oscillator strengths, ${ }^{[31-33]}$ dipole moments ${ }^{[17,32,33]}$ and chemical reactivity. ${ }^{[34-38]}$ Owing to this progress, it is now possible for many different types of molecules and states to establish beforehand the expected accuracy in the calculation of an excitation energy by a given method. Furthermore, because of the availability of efficient computer codes for finding equilibrium geometries of molecules in both ground and excited states, it is in many cases straightforward to calculate both vertical and 0-0 excitation energies, especially for molecules that can be regarded as non-

[a] Dr. J. Wang

Jiangsu Key Laboratory for Chemistry of Low-Dimensional Materials Jiangsu Engineering Laboratory for Environment Functional Materials School of Chemistry and Chemical Engineering Huaiyin Normal University

No. 111 West Changjiang Road, 223300 Huaian, Jiangsu Province (China)

[b] Prof. B. Durbeej

Division of Theoretical Chemistry, IFM

Linköping University

58183 Linköping (Sweden)

E-mail: bodur@ifm.liu.se

Supporting information for this article is available on the WWW under https://doi.org/10.1002/cptc.202100178

of (c) 2021 The Authors. ChemPhotoChem published by Wiley-VCH GmbH. This is an open access article under the terms of the Creative Commons Attribution Non-Commercial NoDerivs License, which permits use and distribution in any medium, provided the original work is properly cited, the use is non-commercial and no modifications or adaptations are made.
13 conjugated double bonds. Employing several different density functional methods, it is shown that thermal fluctuations in the conjugation result in the Boltzmann-weighted average excitation energies for this state being shifted by up to $0.05 \mathrm{eV}$ relative to those obtained from purely static calculations. Accordingly, it is concluded that the effects of thermal motion on excitation energies of conjugated systems can be quite large even for molecules with distinct potential-energy minima. flexible in tending to adopt conformations close to their potential-energy minima. In fact, while vertical energies are computed as energy differences between ground and excited states at ground-state equilibrium geometries, $0-0$ energies are obtained almost as readily as energy differences between the states at their respective equilibrium geometries, with inclusion of zero-point vibrational energy corrections for the two states.

Despite the common use and success of this static computational approach to estimate excitation energies, it is clear that representing molecules exclusively by their equilibrium geometries is an approximation in that thermal motion along internal coordinates is neglected. Indeed, such motion will invariably influence experimentally derived excitation energies with which the calculated values are often compared. Moreover, while this approximation is usually assumed to be good/poor for molecules with small/large conformational flexibility, ${ }^{[39,40]}$ it appears from the existing literature that its quantitative accuracy for non-flexible systems with distinct potential-energy minima has never been thoroughly tested. Therefore, it is the aim of the present work to begin filling this important gap.

To this end, we here consider the carotenoid astaxanthin (AXT, 3,3'-dihydroxy- $\beta$-carotene-4,4'-dione, see Figure 1 ) and use density functional theory (DFT) methods to calculate vertical excitation energies for the strongly absorbing $B_{u}$-like state of this compound both through the traditional static approach and through a dynamical approach where thermal motion is accounted for by means of ab initio molecular dynamics (AIMD) simulations. ${ }^{[41,42]}$

There are at least two reasons why it is of interest to compare these two approaches for the particular case of AXT. First, this orange-red pigment has through the years been the subject of numerous experimental ${ }^{[43-55]}$ and computational studies ${ }^{[48,52,55-62]}$ aimed at clarifying why its UV-Vis absorption attributed to the aforementioned state is shifted from (depend- 


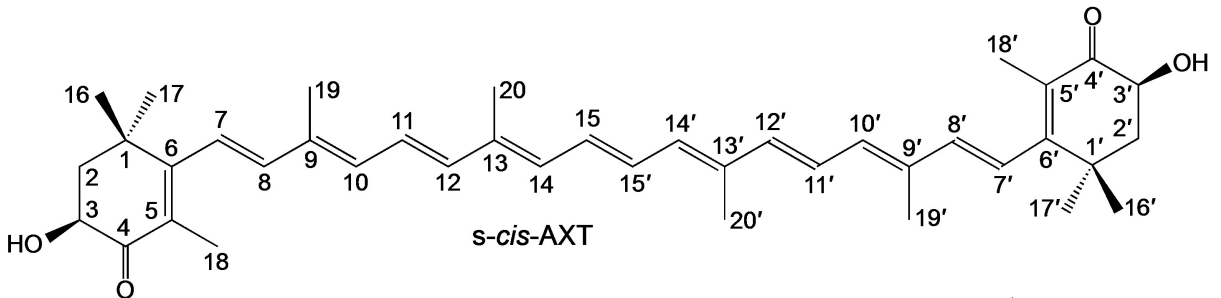<smiles>C[C]1C[C@H](O)C(=O)C(C)=C1/C=C/C(C)=C/C=C/C(C)=C/C=C/C=C(C)/C=C/C=C(C)/C=C/C1=C(C)C(=O)[C@@H](O)C[C@@H]1C</smiles><smiles>CC1=C(/C=C/C(C)=C/C=C/C(C)=C/C=C/C=C(C)/C=C/C=C(C)/C=C/C2=C(C)C(=O)[C@@H](O)CC2(C)C)[C@@H](C)C[C@H](O)C1=O</smiles>

Figure 1. Chemical structure and atom numbering of astaxanthin shown in its s-cis, s-trans and s-trans-s'-cis stereoisomeric forms. The s-cis and s-trans forms are approximately of $\mathrm{C}_{2 \mathrm{~h}}$ symmetry, which is why we herein refer to the strongly absorbing excited state of astaxanthin as a "B-like" state.

ing on the solvent) $\lambda_{\max } \approx 470-500 \mathrm{~nm}$ in dilute solutions, ${ }^{[44,53]}$ to $\lambda_{\max } \approx 630 \mathrm{~nm}$ when it instead occurs in the $\alpha$-crustacyanin protein complex responsible for the characteristic dark-blue coloration of lobster (Homarus gammarus) shell. ${ }^{[45,53]}$ In fact, this bathochromic shift is among the largest protein-induced spectral shifts known and many different explanations for its origin have been put forth and disputed. Generally, the proposed mechanisms pertain to the precise physical arrangement of the two AXT molecules contained in each of the eight dimeric $\beta$-crustacyanin subunits of $\alpha$-crustacyanin, ${ }^{[5,54,61]}$ exciton coupling between the AXT molecules, ${ }^{[44,45,49,52,53]}$ and various ways in which the protein environment polarizes the AXT molecules or even changes their protonation state..$^{[43,46-48,50,54-56,58,61]}$ Despite these efforts, there is still controversy as to which mechanism is key for explaining the bathochromic shift, ${ }^{[55,62]}$ although the current consensus view appears to be that exciton coupling between the proteinbound carotenoids does not play a dominant role. ${ }^{[5,59,60]}$

The second reason why AXT is a suitable system for the present investigation is the high sensitivity of its excitation energies to small changes in the geometry of its 13 conjugated double bonds (see Figure 1) that can be envisioned based on previous studies of other carotenoids and conjugated molecules that also feature a long polyene chain. ${ }^{[63,64]}$ Indeed, for the calculation of excitation energies, it is especially under such circumstances that the reliance on a single potential-energy minimum warrants careful attention. Notably, in the ongoing debate on the crustacyanin coloration mechanism, it has been suggested that one way in which the protein environment controls the UV-Vis absorption is by causing thermal fluctuations in the conjugation pattern of AXT. ${ }^{[62]}$ Accordingly, it becomes pertinent to explore the possible effect of such fluctuations also in the absence of the protein, as done in this work.
Finally, we emphasize that although the presumed strong coupling of the excitation energies of AXT to the bondstretching modes of its polyene chain could also be probed through the calculation of vibrationally resolved absorption spectra, such an undertaking would fall outside the scope of our work, which is to fill an important gap in the existing literature by comparing static and dynamical approaches to the calculation of purely electronic excitation energies. This comparison will provide a long overdue assessment of a key issue in computational photochemistry.

\section{Computational Methods}

Considering all three stereoisomers of AXT that are possible with respect to how the two $\beta$-ionone rings are oriented relative to the C6-C7 and $\mathrm{C6}^{\prime}-\mathrm{C7}^{\prime}$ bonds (s-cis, s-trans and s-trans-s'-cis, see Figure 1), the investigation involved DFT modeling with five different density functionals based on the generalized gradient

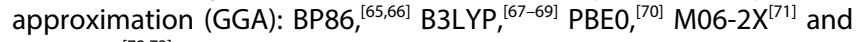
$\omega B 97 X D .^{[72,73]}$ Briefly, BP86 is a pure GGA that lacks exact exchange; B3LYP and PBEO are global hybrid GGAs that contain a fixed fraction of exact exchange ( 20 and $25 \%$, respectively); M06-2X $(54 \%)$ is a global hybrid meta-GGA that includes a dependence on the kinetic energy density; and $\omega B$ 97XD is a range-separated hybrid GGA that contains a variable fraction of exact exchange (22-100\%) depending on the interelectronic distance - smaller at small distances and larger at large distances. Although $\omega B$ 97XD also incorporates empirical atom-atom $1 / R^{6}$ terms $s^{[74]}$ to better account for dispersion interactions, these terms do not impact excitation energies computed with this functional.

In the static approach to the calculation of vertical excitation energies, the ground-state geometries of the AXT isomers were optimized without symmetry constraints using the different density functionals in combination with the $6-31 \mathrm{G}(\mathrm{d}, \mathrm{p})$ double-zeta basis set. This choice of basis set reflects that the geometry optimizations need to be carried out with the same basis set as that 
deemed affordable for the much more expensive AIMD simulations performed in the dynamical approach. However, for comparative purposes, geometry optimizations were also done with a series of larger basis sets, including both double-zeta ones with diffuse functions on all $\mathrm{C} / \mathrm{O}(6-31+\mathrm{G}(\mathrm{d}, \mathrm{p}))$ or $\mathrm{C} / \mathrm{O} / \mathrm{H}(6-31++\mathrm{G}(\mathrm{d}, \mathrm{p}))$ atoms, and triple-zeta ones without $(6-311 \mathrm{G}(\mathrm{d}, \mathrm{p})$ and $6-311+$ $\mathrm{G}(\mathrm{d}, \mathrm{p}))$ and with $(6-311 \mathrm{G}(2 \mathrm{df}, \mathrm{p})$ and $6-311+\mathrm{G}(2 \mathrm{df}, \mathrm{p}))$ additional polarization functions. For further comparative purposes, the geometries of the AXT isomers were also optimized with the $a b$ initio Møller-Plesset second-order perturbation theory (MP2) $)^{[75,76]}$ and approximate coupled-cluster singles and doubles $(\mathrm{CC} 2)^{[77,78]}$ methods. These calculations were done within the resolution-ofthe-identity approximation, ${ }^{[79]}$ employing the SVP double-zeta basis set (which is similar to 6-31G(d,p)) and its auxiliary counterpart for density fitting. ${ }^{[80]}$ Based on the optimized ground-state geometries, which through analytical (DFT) or numerical (MP2 and CC2) frequency calculations were confirmed to be potential-energy minima, vertical excitation energies for the strongly absorbing $B_{u^{-}}$ like state were obtained by performing singlepoint calculations using the same aforementioned set of density functionals and the $6-31 G(d, p)$ basis set in the framework of time-dependent DFT (TDDFT). ${ }^{[81-84]}$ In these calculations, the SMD method ${ }^{[85]}$ was used to provide a continuum-model description of $n$-hexane, which is one solvent in which the UV-Vis absorption of AXT has been recorded. ${ }^{[44,53]}$

Although of minor importance for a study that focuses exclusively on the strongly absorbing $\mathrm{B}_{\mathrm{u}}$-like excited state of AXT, which has $\pi \pi^{*}$ character and is ideally suited for TD-DFT calculations, it should be noted that TD-DFT generally predicts that this state is the lowest excited state $\left(S_{1}\right) \cdot{ }^{[59]}$ Accordingly, the corresponding vertical excitation energy is here denoted $\Delta E\left(\mathrm{~S}_{1}\right)$. However, for linear polyenes with more than three double bonds, it is well known that the lowest excited state is a dark $A_{g}$ state, ${ }^{[86,87]}$ which has significant double-excitation character ${ }^{[88]}$ and therefore poses an appreciable challenge for standard TD-DFT and other singlereference methodologies. ${ }^{[89]}$ In this connection, and in light of the "ghost-state" problem oftentimes facing TD-DFT calculations with pure functionals or functionals containing a small fraction of exact exchange ${ }^{[00]}$ it should be emphasized that all five functionals employed herein do identify the bright $B_{u}$-like state of AXT as $S_{1}$, and that no lower-lying, artificial ghost states without an experimental counterpart were found in any of the calculations.

In the dynamical approach to the calculation of vertical excitation energies, in turn, the use of geometry optimizations to derive equilibrium structures was superseded by the use of AIMD simulations to instead generate an ensemble of ground-state geometries for each of the three AXT isomers. Employing the atom-centered density matrix propagation (ADMP) MD model, ${ }^{[91,92]}$ the simulations were carried out with the same set of density functionals and the same basis set $(6-31 \mathrm{G}(\mathrm{d}, \mathrm{p}))$ as the previous geometry optimizations. Using an integration time step of $0.1 \mathrm{fs}$, which is about two orders of magnitude smaller than the fastest time scale $(\sim 10 \mathrm{fs})$ on which thermal fluctuations in the conjugation are expected to occur, ${ }^{[93]}$ the simulations were run at a temperature of $298 \mathrm{~K}$ for a total of $20 \mathrm{ps}$. Of these, the first $10 \mathrm{ps}$ were considered as equilibration. For each trajectory, an ensemble of 100 different AXT geometries were then collected by extracting one structure from the subsequent 10-ps "production" trajectory every $100 \mathrm{fs}$, whereafter $\Delta E\left(\mathrm{~S}_{1}\right)$ energies for the resulting ensembles were calculated at the same level of theory as in the static approach, using TD-BP86 for BP86 geometries, TD-B3LYP for B3LYP geometries, and so on. Based on these results, for each ensemble an estimate of the average $\Delta E\left(\mathrm{~S}_{1}\right)$ energy, here denoted $\left\langle\Delta E\left(S_{1}\right)\right\rangle$, that includes the effect of thermal motion was obtained as (the Boltzmann-weighted average at $298 \mathrm{~K}$ ) [Eq. (1)]:
$\left\langle\Delta E\left(S_{1}\right)\right\rangle=\frac{\sum_{i}^{100} \Delta E_{i}\left(\mathrm{~S}_{1}\right) e^{-E_{i}\left(S_{0}\right) / k_{B} T}}{\sum_{i}^{100} e^{-E_{i}\left(S_{0}\right) / k_{B} T}}$

where $\Delta E_{i}\left(\mathrm{~S}_{1}\right)$ is the vertical excitation energy for geometry $i$ in the ensemble and $E_{i}\left(\mathrm{~S}_{0}\right)$ is the ground-state energy of that geometry relative to the most stable geometry in the ensemble.

The MP2 and CC2 calculations were done with the RICC2 module ${ }^{[94]}$ of the TURBOMOLE 6.3 suite of programs. ${ }^{[95,96]}$ All other calculations were done with the Gaussian 16 suite of programs. ${ }^{[97]}$

\section{Results and Discussion}

\subsection{Static Calculations}

Our first order of business will be to assess how well the $\Delta E\left(\mathrm{~S}_{1}\right)$ energies calculated through the static approach reproduce the experimental $\Delta E\left(\mathrm{~S}_{1}\right)$ energy that can be derived from the $\lambda_{\max }$ of the UV-Vis absorption spectrum of AXT in $n$-hexane ${ }^{[44]}$ the solvent considered in the calculations. This is done in Table 1, wherein the experimental value of $2.63 \mathrm{eV}$ is associated with the s-cis isomer, because this isomer is known to be dominant in solution. ${ }^{[98,99]}$ Focusing particularly on the energies obtained when one and the same density functional is used for both geometry optimization and $\Delta E\left(\mathrm{~S}_{1}\right)$ singlepoint calculation, it can be seen that the spread in the $\Delta E\left(\mathrm{~S}_{1}\right)$ values for s-cis-AXT, as well as for the other isomers, is large, ranging from $1.47 \mathrm{eV}$ at the BP86 level to 1.91, 2.01, 2.62 and $2.74 \mathrm{eV}$ at the B3LYP, PBE0, M06-2X and $\omega B 97 X D$ levels, respectively. Furthermore, from Table S1 of the Supporting Information (SI), it is clear that these results are not very sensitive to which basis set (here $6-31 G(d, p))$ is used for the geometry optimizations. The observation that the pure BP86 functional yields the smallest $\Delta E\left(\mathrm{~S}_{1}\right)$ energy, well below the experimental value, and that the inclusion of successively larger fractions of exact exchange by the global hybrid functionals leads to successively larger $\Delta E\left(\mathrm{~S}_{1}\right)$ energies, is consistent with the typical behavior of these methods when applied to organic molecules. ${ }^{[2]}$ The finding that M06-2X (54\%) comes much closer than B3LYP (20\%) and PBE0 $(25 \%)$ to the experimental value may reflect that the fraction of exact exchange needed in a global hybrid for it to perform optimally for AXT is greater than the commonly advocated value of $\sim 20 \%{ }^{[2]}$ on the grounds that AXT is a relatively large chromophore. $^{[4]}$

As for the calculated $\Delta E\left(\mathrm{~S}_{1}\right)$ energies of s-trans-s'-cis-AXT and s-trans-AXT, in turn, they are overall quite similar to those of s-cis-AXT, consistently being red-shifted by $0.02-0.06$ and $0.03-0.10 \mathrm{eV}$, respectively, depending on which functional is used. This is a straightforward consequence of the fact that an s-trans orientation of one (in s-trans-s'-cis-AXT) or both (in strans-AXT) of the $\beta$-ionone rings relative to the polyene chain enables better conjugation of all 13 double bonds of the molecule than afforded when both rings have an s-cis orientation (in s-cis-AXT). Specifically, as can be inferred from Figure 2, an s-trans orientation allows for a higher degree of coplanarity of the rings (that have $2+2$ double bonds) with the polyene chain (that has 9 double bonds), as the ensuing steric 
Table 1. Vertical excitation energies $\left(\triangle E\left(\mathrm{~S}_{1}\right)\right.$, in eV) of AXT and the corresponding oscillator strengths ( $f$ ) calculated with different DFT methods at different molecular geometries, bond-length alternation (BLA, in $\AA$ ) in these geometries, and maximal absolute variation in $\triangle E\left(\mathrm{~S}_{1}\right)$ and $B L A$ values between the geometries $\left(|\Delta|_{\max }\right)$.

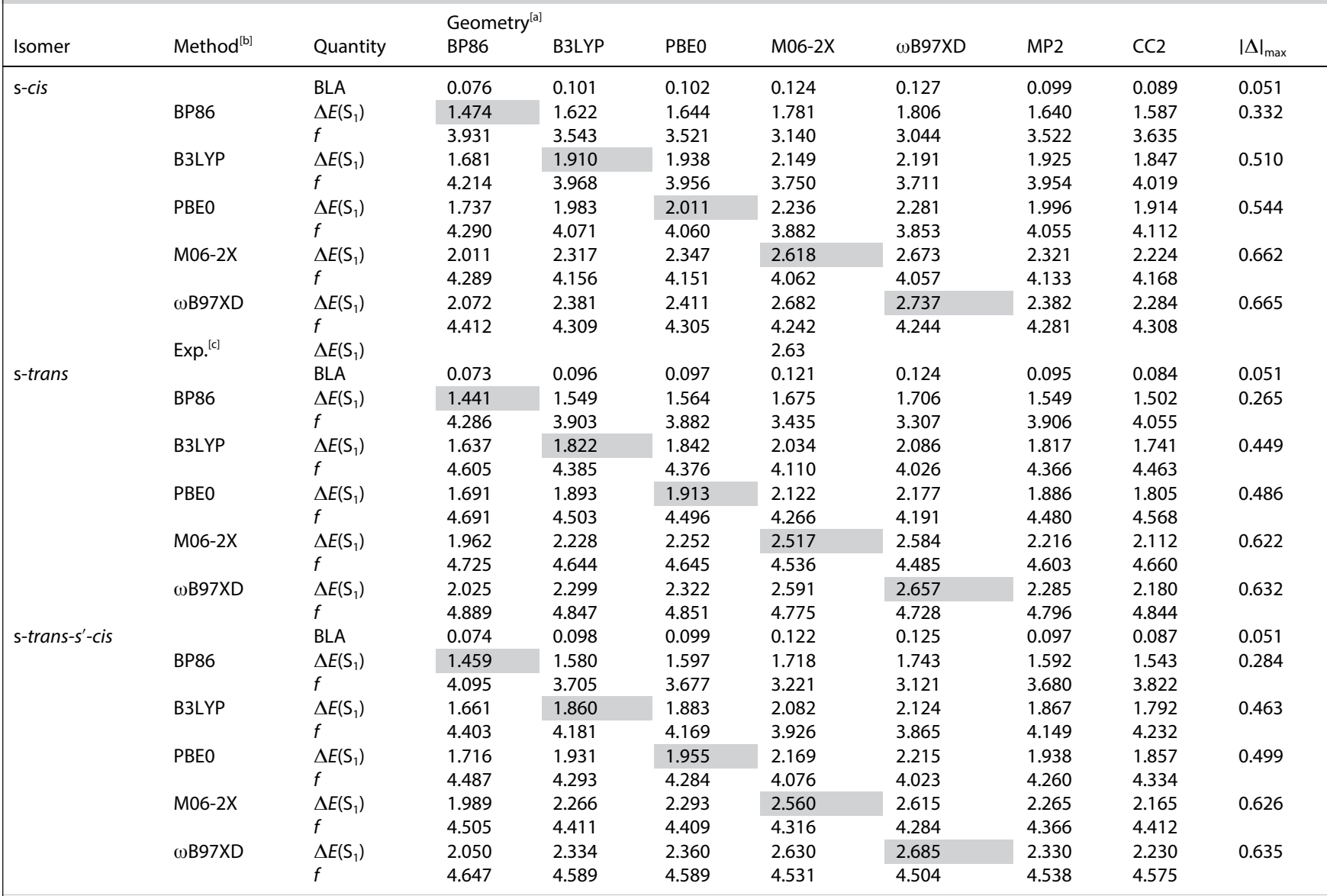

[a] Molecular geometries optimized with the 6-31G(d,p) (DFT) or the SVP (MP2 and CC2) basis set. [b] Excitation energies calculated with the 6-31G(d,p) basis set. [c] Experimental value in $n$-hexane derived from the $\lambda_{\max }$ of $472 \mathrm{~nm}$ given in Ref. [44].

repulsion between the $\mathrm{C} 1$ methyl groups and the polyene chain is smaller than that between the $C 5$ methyl groups and the polyene chain in s-cis-AXT.

Further scrutiny of the results in Table 1 reveals that the calculated $\Delta E\left(\mathrm{~S}_{1}\right)$ energies throughout are extremely sensitive to the molecular geometry. In fact, they vary by up to $0.27-0.33$ (BP86), 0.45-0.51 (B3LYP), 0.49-0.54 (PBE0), 0.62-0.66 (M06-2X) and $0.63-0.67 \mathrm{eV}(\omega \mathrm{B} 97 \mathrm{XD})$, depending on at which geometry (BP86, B3LYP, PBE0, M06-2X, $\omega B$ 97XD, MP2 or CC2) the calculations are performed. Moreover, without exception, the $\Delta E\left(\mathrm{~S}_{1}\right)$ values increase with the fraction of exact exchange included in the functional used to optimize the molecular geometries. For example, for s-cis-AXT, the M06-2X energy is $2.01 \mathrm{eV}$ at the BP86 geometry, but increases to 2.32, 2.35, 2.62 and $2.67 \mathrm{eV}$ at the B3LYP, PBEO, M06-2X and $\omega B$ 97XD geometries, respectively. In order to better understand this trend, Table 1 also provides values of the bond-length alternation (BLA) in the different geometries, which is a quantity defined as the difference between the average length of the single bonds and the average length of the double bonds along the conjugated chain in question (for AXT, there are 13 double and 12 single bonds to consider, see Figure 1). As can be seen, the
BLA is the smallest in the BP86 geometries (0.073-0.076 $\AA$ ), but gradually grows larger in the B3LYP $(0.096-0.101 \AA)$, PBEO

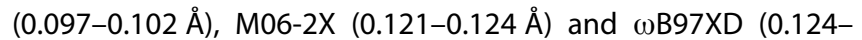
$0.127 \AA$ ) geometries. This is consistent with the general tendency that pure functionals and the Hartree-Fock method (which provides an exact treatment of exchange) show opposing behavior by under- and overestimating, respectively, the BLA in conjugated systems. Owing to a cancellation of these two effects and based on previous studies of other conjugated systems, ${ }^{[63,100]}$ it seems likely that the BLA predicted by B3LYP and PBEO is the most accurate one. This notion is supported by the finding in Table 1 that these values (0.096$0.102 \AA$ ) are in quite close agreement with those predicted by MP2 and CC2 (0.084-0.099 $\AA$ ). Furthermore, it can be envisioned that the $20-25 \%$ fractions of exact exchange included in B3LYP and PBEO do well in offsetting the self-interaction error present in all density functionals in common use, ${ }^{[90,101,102]}$ while keeping some of the undesirable features of Hartree-Fock theory at bay. This error, which is particularly pronounced for pure functionals, ${ }^{[90,101,102]}$ is responsible for the tendency of these methods to favor delocalized bonding patterns. 

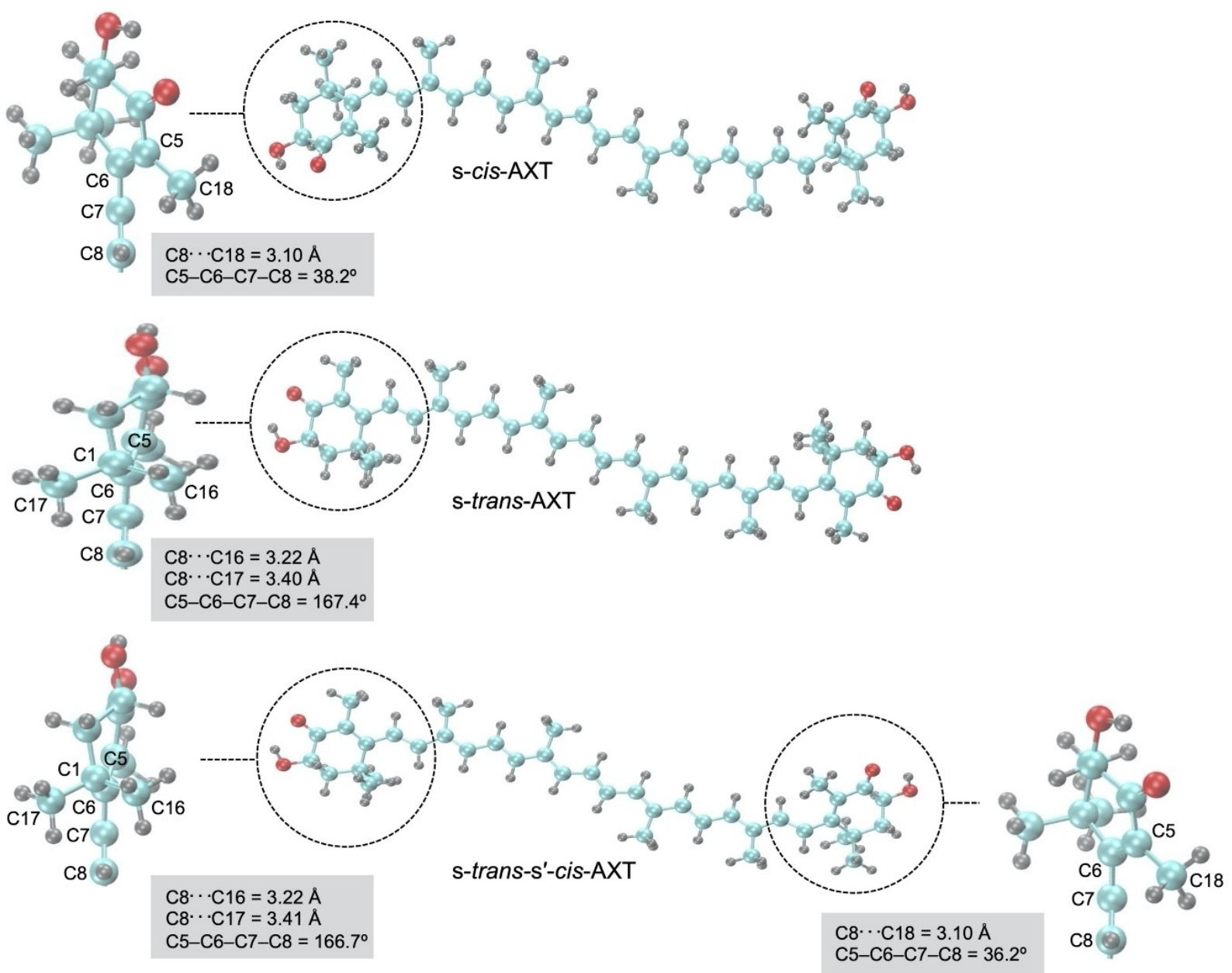

Figure 2. B3LYP/6-31G(d,p)-optimized structures of s-cis-AXT, s-trans-AXT and s-trans-s'-cis-AXT. The insets show geometric parameters that reflect the degrees of co-planarity (the $\mathrm{C} 5-\mathrm{C} 6-\mathrm{C} 7-\mathrm{C} 8$ dihedral angle) and steric repulsion (the C8 $\cdots \mathrm{C} 16, \mathrm{C} 8 \cdots \mathrm{C} 17$ and $\mathrm{C} 8 \cdots \mathrm{C} 18$ distances) between the $\beta$-ionone rings and the polyene chain.

While BLA thus is found to offer a clear qualitative explanation for the sensitivity of the $\Delta E\left(\mathrm{~S}_{1}\right)$ energies in Table 1 to the molecular geometry, it is also of interest to explore this issue more quantitatively. This is done in Figure 3, wherein the $\triangle E\left(\mathrm{~S}_{1}\right)$ energies are plotted as a function of BLA. Remarkably, by subjecting the data to regression analyses, for each of the three isomers near-perfect linear relationships between the two quantities could be established for all five of the density functionals, with the smallest associated $R^{2}$-value amounting to 0.988. Notably, although the concept of BLA has been invoked to explain photophysical properties of many different systems, ${ }^{[64,103-105]}$ studies demonstrating a similarly strong dependency of these properties on BLA appear to be rare. Furthermore, from the resulting slopes of the plots, which vary from 5.0-6.3 eV $\AA^{-1}$ for BP86 to 11.9-12.6 eV $\AA^{-1}$ for $\omega B$ 97XD, it is clear that even a small difference in the BLA between two AXT geometries will have a distinct effect on the calculated $\Delta$ $E\left(S_{1}\right)$ energies. For example, it suffices that this difference equals $\sim 0.016 \AA$ for the effect to range in magnitude from $\sim 0.1 \mathrm{eV}$ at the BP86 level to $\sim 0.2 \mathrm{eV}$ at the $\omega \mathrm{B} 97 \mathrm{XD}$ level. It is precisely this sensitivity that makes AXT an ideal system for comparing static and dynamical approaches to the calculation of vertical excitation energies, which we turn to next.

\subsection{Comparison Static and Dynamical Calculations}

The $\left\langle\Delta E\left(S_{1}\right)\right\rangle$ energies calculated through the dynamical approach are presented in Figure $4 \mathrm{a}$, alongside the corresponding static $\triangle E\left(\mathrm{~S}_{1}\right)$ energies. For further clarity, the absolute differences $\left|\Delta E\left(S_{1}\right)-\left\langle\Delta E\left(S_{1}\right)\right\rangle\right|$ between the two quantities are highlighted in Figure $4 \mathrm{~b}$. However, before discussing these results, it should be noted that the $\left\langle\Delta E\left(S_{1}\right)\right\rangle$ energies, derived by averaging over the full production trajectories, are consistently well converged with respect to estimates of $\left\langle\Delta E\left(S_{1}\right)\right\rangle$ obtained by averaging over shorter trajectories (see Figure $\mathrm{S} 1$ of the $\mathrm{SI}$ ). Moreover, of technical significance, the total energies along the production trajectories are well conserved (see Figure $\mathrm{S} 2$ of the $\mathrm{SI}$ ), which indicates that the integration time step of $0.1 \mathrm{fs}$ in the AIMD simulations is sufficiently small.

Notably, from Figure $4 \mathrm{~b}$, it is clear that the $\left|\Delta E\left(S_{1}\right)-\left\langle\Delta E\left(S_{1}\right)\right\rangle\right|$ values can be quite large and that, 

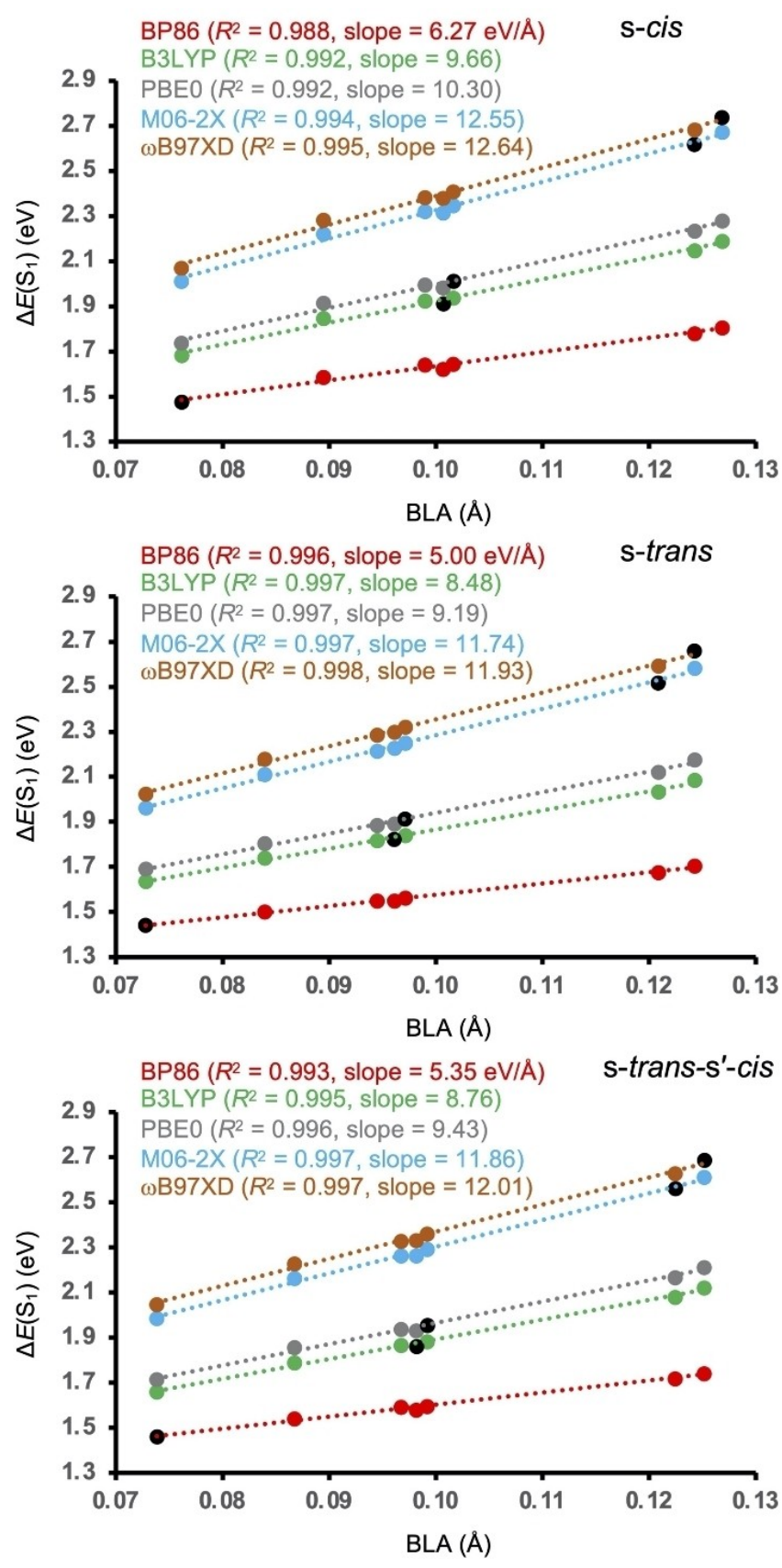

Figure 3. $\Delta E\left(\mathrm{~S}_{1}\right)$ energies of s-cis-AXT, s-trans-AXT and s-trans-s'-cis-AXT calculated with different DFT methods at molecular geometries with different BLA. Data points for which the calculations are done using geometries optimized with the method in question are colored black.

consequently, the effects of thermal motion cannot generally be ignored. In fact, in some cases they are as large as $0.05 \mathrm{eV}$ and for all density functionals except B3LYP, the mean $\left|\Delta E\left(S_{1}\right)-\left\langle\Delta E\left(S_{1}\right)\right\rangle\right|$ value over the three isomers is $0.03-$ $0.04 \mathrm{eV}$ (for B3LYP, this value is $0.01 \mathrm{eV}$ ). Hence, the influence of thermal motion on the calculated excitation energies of AXT is evident, despite that AXT exhibits distinct potential-energy minima and, as such, has been the subject of numerous computational studies in which its strongly absorbing $\mathrm{B}_{\mathrm{u}}$-like state is described by the traditional, static quantum chemical

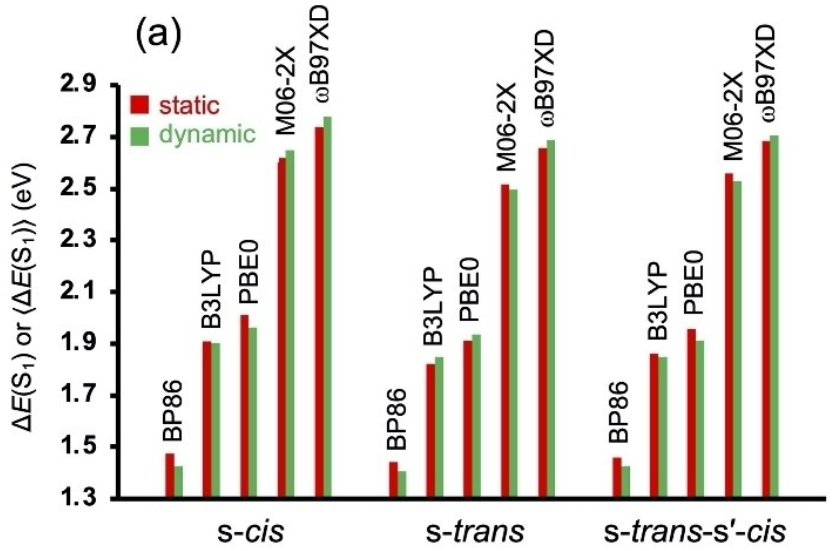

(b)

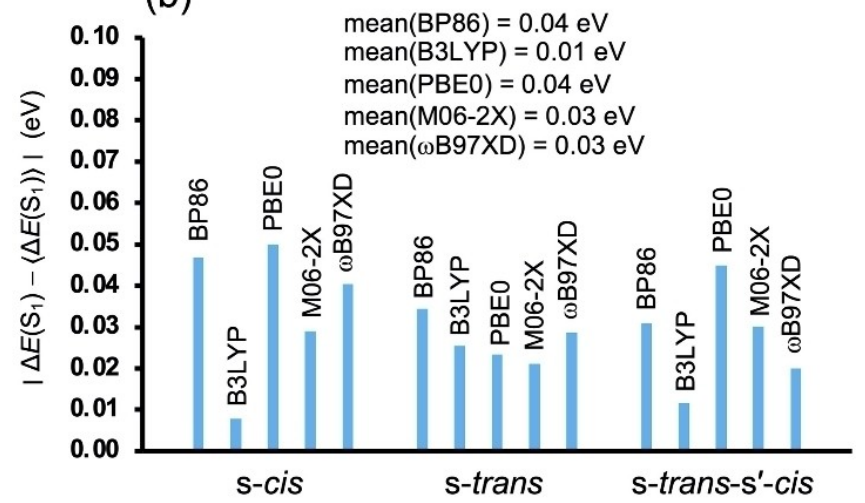

Figure 4. (a) Comparison of static $\Delta E\left(\mathrm{~S}_{1}\right)$ and dynamical $\left\langle\Delta E\left(S_{1}\right)\right\rangle$ energies of $\mathrm{s}$-cis-AXT, s-trans-AXT and s-trans-s'-cis-AXT calculated with different DFT methods. (b) Absolute differences between the $\Delta E\left(\mathrm{~S}_{1}\right)$ and $\left\langle\Delta E\left(S_{1}\right)\right\rangle$ energies. Indicated are also the mean values (over the three isomers) of the absolute differences for the different methods.

approach. ${ }^{[48,52,55-61]}$ Furthermore, although an effect of $\sim 0.05 \mathrm{eV}$ is well tolerable in many situations where calculated excitation energies are used to predict and assess chemical phenomena, this is not a small shift in a spectroscopic context or in comparison with many other factors that influence computational accuracy and generally are being paid much more attention, such as the choice of basis set. For example, as already seen in Table S1 or found in a previous study, ${ }_{1}^{[55]}$ the sensitivity of the static $\Delta E\left(\mathrm{~S}_{1}\right)$ energies of AXT to the use of a larger basis set than $6-31 \mathrm{G}(\mathrm{d}, \mathrm{p})$ for ground-state geometry optimization (Table S1) or excited-state singlepoint energy calculation $^{[55]}$ does not exceed $0.05 \mathrm{eV}$. Moreover, it should be pointed out that an effect of $\sim 0.05 \mathrm{eV}$ is rather large in relation to the "chemical accuracy" threshold often sought for calculated excitation energies, ${ }^{[90]}$ which by some researchers is set at $1 \mathrm{kcal} \mathrm{mol}^{-1} \approx 0.04 \mathrm{eV}^{[5,11]}$ whereas others prefer $0.1 \mathrm{eV}^{[106]}$ At the same time, the effect is small compared to the average errors that all density functionals, including accurate double hybrids ones, ${ }^{[90,107-109]}$ show for excitation energies. Thus, the effect is not likely to be the main source of error when such calculations are performed in a static fashion.

Altogether, the results of this work show that caution should be exercised when excitation energies of conjugated molecular systems are calculated based exclusively on their 
equilibrium geometries and, particularly, that such caution is warranted also when the molecules in question have distinct potential-energy minima. Given that our work appears to be the first to systematically explore this issue and has been carried out with a strict focus on AXT - partly because AXT features a polyene chain and partly because it is not feasible to subject a plethora of molecules to expensive AIMD simulations with different density functionals - it will be a task for future benchmark studies to investigate to what extent the current results also apply to other types of molecules.

It is also worthwhile to briefly comment on the present results in light of proposed mechanisms ${ }^{[43-62]}$ for the tuning of the UV-Vis absorption of AXT by crustacyanin proteins. In fact, as noted in the Introduction, one such mechanism involves protein-induced thermal fluctuations in the conjugation of

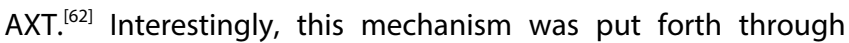
DFT-based AIMD simulations akin to the present ones but considering AXT in a $\beta$-crustacyanin protein environment. ${ }^{[62]}$ Thereby, a $\left|\Delta E\left(S_{1}\right)-\left\langle\Delta E\left(S_{1}\right)\right\rangle\right|$ value of $\sim 0.20 \mathrm{eV}$ was reported, which is much larger than that of $\sim 0.05 \mathrm{eV}$ documented herein for an isolated AXT molecule. Accordingly, another suitable task for future computational work will be to elucidate exactly how this strengthening of the thermal fluctuations by the protein comes about.

\section{Conclusions}

In summary, using five different density functionals (BP86, B3LYP, PBE0, M06-2X and $\omega B$ 97XD), we have compared static and dynamical approaches to the calculation of vertical excitation energies for the optically bright state of AXT, a carotenoid that is both of fundamental (for the coloration of crustacyanin proteins ${ }^{[51]}$ ) and applied (as a mechanosensitive fluorescent probe ${ }^{[110]}$ or a nutraceutical ${ }^{[111]}$ ) interest. First, from the static calculations, which neglect the effects of thermal motion and are performed based on equilibrium structures obtained from geometry optimizations, it is established that the resulting excitation energies are very sensitive (typically by $0.5 \mathrm{eV}$ or more) to the geometry and exhibit near-perfect linear relationships ( $R^{2}$-values of 0.988 or larger) with the BLA in the conjugated carotenoid backbone. Subsequently, when these energies are compared with Boltzmann-weighted average energies obtained from the dynamical calculations, which account for thermal fluctuations in the conjugation and are carried out based on an ensemble of structures generated by AIMD simulations, such fluctuations are found to shift the energies by up to $0.05 \mathrm{eV}$. Based on this result, we conclude that the effects of thermal motion on excitation energies of conjugated systems can be quite large even for molecules with distinct potential-energy minima for which static calculations are otherwise the norm.

\section{Acknowledgments}

This work was supported by the Olle Engkvist Foundation (grants 184-568 and 204-0183), the Swedish Research Council (grant 2019-03664), ÅForsk (grant 20-570) and the Carl Trygger Foundation (grant CTS 20:102). The calculations were enabled by resources provided by (a) the Swedish National Infrastructure for Computing at the National Supercomputer Centre partially funded by the Swedish Research Council (grant 2018-05973) and (b) the National Supercomputer Centre funded by Linköping University.

\section{Conflict of Interest}

The authors declare no conflict of interest.

Keywords: Bond-length alternation - Carotenoids

Conjugation • Density functional calculations - Molecular dynamics

[1] M. Schreiber, M. R. Silva-Junior, S. P. A. Sauer, W. Thiel, J. Chem. Phys. 2008, 128, 134110.

[2] D. Jacquemin, V. Wathelet, E. A. Perpète, C. Adamo, J. Chem. Theory Comput. 2009, 5, 2420-2435.

[3] M. Caricato, G. W. Trucks, M. J. Frisch, K. B. Wiberg, J. Chem. Theory Comput. 2010, 6, 370-383.

[4] L. Goerigk, S. Grimme, J. Chem. Phys. 2010, 132, 184103.

[5] R. Send, M. Kühn, F. Furche, J. Chem. Theory Comput. 2011, 7, 23762386.

[6] D. Jacquemin, A. Planchat, C. Adamo, B. Mennucci, J. Chem. Theory Comput. 2012, 8, 2359-2372.

[7] N. O. C. Winter, N. K. Graf, S. Leutwyler, C. Hättig, Phys. Chem. Chem. Phys. 2013, 15, 6623-6630.

[8] C. Fang, B. Oruganti, B. Durbeej, J. Phys. Chem. A 2014, 118, 41574171.

[9] D. Jacquemin, I. Duchemin, X. Blasé, J. Chem. Theory Comput. 2015, 11, 5340-5359.

[10] B. Oruganti, C. Fang, B. Durbeej, Mol. Phys. 2016, 114, 3448-3463.

[11] P.-F. Loos, N. Galland, D. Jacquemin, J. Phys. Chem. Lett. 2018, 9, 46464651.

[12] P.-F. Loos, D. Jacquemin, ChemPhotoChem 2019, 3, 684-696.

[13] A. Ottochian, C. Morgillo, I. Ciofini, M. J. Frisch, G. Scalmani, C. Adamo, J. Comput. Chem. 2020, 41, 1242-1251.

[14] P.-F. Loos, F. Lipparini, M. Boggio-Pasqua, A. Scemama, D. Jacquemin, J. Chem. Theory Comput. 2020, 16, 1711-1741.

[15] P.-F. Loos, A. Scemama, D. Jacquemin, J. Phys. Chem. Lett. 2020, 11, 2374-2383.

[16] X. Blasé, I. Duchemin, D. Jacquemin, P.-F. Loos, J. Phys. Chem. Lett. 2020, 11, 7371-7382.

[17] F. Furche, R. Ahlrichs, J. Chem. Phys. 2002, 117, 7433-7447.

[18] C. A. Guido, S. Knecht, J. Kongsted, B. Mennucci, J. Chem. Theory Comput. 2013, 9, 2209-2220.

[19] R. Guareschi, C. Filippi, J. Chem. Theory Comput. 2013, 9, 5513-5525.

[20] D. Tuna, Y. Lu, A. Koslowski, W. Thiel, J. Chem. Theory Comput. 2016, $12,4400-4422$.

[21] Š. Budzák, G. Scalmani, D. Jacquemin, J. Chem. Theory Comput. 2017, 13, 6237-6252.

[22] E. Brémond, M. Savarese, C. Adamo, D. Jacquemin, J. Chem. Theory Comput. 2018, 14, 3715-3727.

[23] J. Wang, B. Durbeej, J. Comput. Chem. 2020, 41, 1718-1729.

[24] R. Grotjahn, M. Kaupp, J. Chem. Theory Comput. 2020, 16, 5821-5834.

[25] M. Sokolov, B. M. Bold, J. J. Kranz, S. Höfener, T. A. Niehaus, M. Elstner, J. Chem. Theory Comput. 2021, 17, 2266-2282.

[26] M. Dierksen, S. Grimme, J. Chem. Phys. 2004, 120, 3544-3554.

[27] T. Petrenko, F. Neese, J. Chem. Phys. 2007, 127, 164319. 
[28] V. Barone, J. Bloino, M. Biczysko, F. Santoro, J. Chem. Theory Comput. 2009, 5, 540-554.

[29] A. Charaf-Eddin, A. Planchat, B. Mennucci, C. Adamo, D. Jacquemin, J. Chem. Theory Comput. 2013, 9, 2749-2760.

[30] F. Santoro, D. Jacquemin, WIREs Comput. Mol. Sci. 2016, 6, 460-486.

[31] M. Caricato, G. W. Trucks, M. J. Frisch, K. B. Wiberg, J. Chem. Theory Comput. 2011, 7, 456-466.

[32] A. Chrayteh, A. Blondel, P.-F. Loos, D. Jacquemin, J. Chem. Theory Comput. 2021, 17, 416-438.

[33] R. Sarkar, M. Boggio-Pasqua, P.-F. Loos, D. Jacquemin, J. Chem. Theory Comput. 2021, 17, 1117-1132.

[34] O. Falklöf, B. Durbeej, J. Comput. Chem. 2014, 35, 2184-2194.

[35] S. Gozem, F. Melaccio, A. Valentini, M. Filatov, M. Huix-Rotllant, N. Ferré, L. M. Frutos, C. Angeli, A. I. Krylov, A. A. Granovsky, R. Lindh, M. Olivucci, J. Chem. Theory Comput. 2014, 10, 3074-3084.

[36] D. Tuna, D. Lefrancois, Ł. Wolański, S. Gozem, I. Schapiro, T. Andruniów, A. Dreuw, M. Olivucci, J. Chem. Theory Comput. 2015, 11, 5758-5781.

[37] M. Boggio-Pasqua, M. J. Bearpark, ChemPhotoChem 2019, 3, 763-769.

[38] C. Fang, B. Durbeej, J. Phys. Chem. A 2019, 123, 8485-8495.

[39] J. Sjöqvist, M. Linares, M. Lindgren, P. Norman, Phys. Chem. Chem. Phys. 2011, 13, 17532-17542.

[40] J. Sjöqvist, R. C. González-Cano, J. T. L. Navarrete, J. Casado, M. C. R. Delgado, M. Linares, P. Norman, Phys. Chem. Chem. Phys. 2014, 16, 24841-24852.

[41] R. Car, M. Parrinello, Phys. Rev. Lett. 1985, 55, 2471-2474.

[42] D. Marx, J. Hutter, Ab Initio Molecular Dynamics: Basic Theory and Advanced Methods, Cambridge University Press, Cambridge, 2009.

[43] R. Kuhn, N. A. Sörensen, Angew. Chem. 1938, 51, 465-466.

[44] M. Buchwald, W. P. Jencks, Biochemistry 1968, 7, 834-843.

[45] M. Buchwald, W. P. Jencks, Biochemistry 1968, 7, 844-859.

[46] V. R. Salares, N. M. Young, H. J. Bernstein, P. R. Carey, Biochim. Biophys. Acta 1979, 576, 176-191.

[47] R. J. Weesie, D. Askin, F. J. H. M. Jansen, H. J. M. de Groot, J. Lugtenburg, G. Britton, FEBS Lett. 1995, 362, 34-38.

[48] R. J. Weesie, F. J. H. M. Jansen, J. C. Merlin, J. Lugtenburg, G. Britton, H. J. M. de Groot, Biochemistry 1997, 36, 7288-7296.

[49] G. Britton, R. J. Weesie, D. Askin, J. D. Warburton, L. Gallardo-Guerrero, F. J. Jansen, H. J. M. de Groot, J. Lugtenburg, J.-P. Cornard, J.-C. Merlin, Pure Appl. Chem. 1997, 69, 2075-2084.

[50] S. Krawczyk, G. Britton, Biochim. Biophys. Acta 2001, 1544, 301-310.

[51] M. Cianci, P. J. Rizkallah, A. Olczak, J. Raftery, N. E. Chayen, P. F. Zagalsky, J. R. Helliwell, Proc. Natl. Acad. Sci. USA 2002, 99, 9795-9800.

[52] A. A. C. van Wijk, A. Spaans, N. Uzunbajakava, C. Otto, H. J. M. de Groot, J. Lugtenburg, F. Buda, J. Am. Chem. Soc. 2005, 127, 1438-1445.

[53] R. P. Ilagan, R. L. Christensen, T. W. Chapp, G. N. Gibson, T. Pascher, T. Polívka, H. A. Frank, J. Phys. Chem. A 2005, 109, 3120-3127.

[54] N. Christensson, K. Žídek, N. C. M. Magdaong, A. M. LaFountain, H. A. Frank, D. Zigmantas, J. Phys. Chem. B 2013, 117, 11209-11219.

[55] S. Begum, M. Cianci, B. Durbeej, O. Falklöf, A. Hädener, J. R. Helliwell, M. Helliwell, A. C. Regan, C. I. F. Watt, Phys. Chem. Chem. Phys. 2015, $17,16723-16732$

[56] B. Durbeej, L. A. Eriksson, Chem. Phys. Lett. 2003, 375, 30-38.

[57] B. Durbeej, L. A. Eriksson, Phys. Chem. Chem. Phys. 2004, 6, 4190-4198.

[58] B. Durbeej, L. A. Eriksson, Phys. Chem. Chem. Phys. 2006, 8, 4053-4071.

[59] A. Strambi, B. Durbeej, J. Phys. Chem. B 2009, 113, 5311-5317.

[60] J. Neugebauer, J. Veldstra, F. Buda, J. Phys. Chem. B 2011, 115, 32163225.

[61] A. P. Gamiz-Hernandez, I. N. Angelova, R. Send, D. Sundholm, V. R. I. Kaila, Angew. Chem. Int. Ed. 2015, 54, 11564-11566; Angew. Chem. 2015, 127, 11726-11729.

[62] D. Loco, F. Buda, J. Lugtenburg, B. Mennucci, J. Phys. Chem. Lett. 2018, 9, 2404-2410.

[63] M. Wanko, M. Hoffmann, P. Strodel, A. Koslowski, W. Thiel, F. Neese, T. Frauenheim, M. Elstner, J. Phys. Chem. B 2005, 109, 3606-3615.

[64] S. Knecht, C. M. Marian, J. Kongsted, B. Mennucci, J. Phys. Chem. B 2013, 117, 13808-13815.

[65] J. P. Perdew, Phys. Rev. B 1986, 33, 8822-8824.

[66] A. D. Becke, Phys. Rev. A 1988, 38, 3098-3100.

[67] C. Lee, W. Yang, R. G. Parr, Phys. Rev. B 1988, 37, 785-789.

[68] A. D. Becke, J. Chem. Phys. 1993, 98, 5648-5652.

[69] P. J. Stephens, F. J. Devlin, C. F. Chabalowski, M. J. Frisch, J. Phys. Chem. 1994, 98, 11623-11627.

[70] C. Adamo, V. Barone, J. Chem. Phys. 1999, 110, 6158-6170.

[71] Y. Zhao, D. G. Truhlar, Theor. Chem. Acc. 2008, 120, 215-241.
[72] J.-D. Chai, M. Head-Gordon, J. Chem. Phys. 2008, 128, 084106

[73] J.-D. Chai, M. Head-Gordon, Phys. Chem. Chem. Phys. 2008, 10, 66156620.

[74] S. Grimme, J. Comput. Chem. 2006, 27, 1787-1799.

[75] F. Weigend, M. Häser, Theor. Chem. Acc. 1997, 97, 331-340.

[76] C. Hättig, A. Hellweg, A. Köhn, Phys. Chem. Chem. Phys. 2006, 8, 11591169.

[77] O. Christiansen, H. Koch, P. Jørgensen, Chem. Phys. Lett. 1995, 243, 409-418.

[78] C. Hättig, J. Chem. Phys. 2003, 118, 7751-7761.

[79] O. Vahtras, J. Almlöf, M. W. Feyereisen, Chem. Phys. Lett. 1993, 213, 514-518.

[80] F. Weigend, M. Häser, H. Patzelt, R. Ahlrichs, Chem. Phys. Lett. 1998, 294, 143-152.

[81] E. Runge, E. K. U. Gross, Phys. Rev. Lett. 1984, 52, 997-1000.

[82] M. A. L. Marques, E. K. U. Gross, Annu. Rev. Phys. Chem. 2004, 55, 427455.

[83] A. Dreuw, M. Head-Gordon, Chem. Rev. 2005, 105, 4009-4037.

[84] M. E. Casida, M. Huix-Rotllant, Annu. Rev. Phys. Chem. 2012, 63, 287323.

[85] A. V. Marenich, C. J. Cramer, D. G. Truhlar, J. Phys. Chem. B 2009, 113, 6378-6396.

[86] B. E. Kohler, Chem. Rev. 1993, 93, 41-54.

[87] M. J. Llansola-Portoles, A. A. Pascal, B. Robert, J. R. Soc. Interface 2017 $14,20170504$.

[88] K. Schulten, M. Karplus, Chem. Phys. Lett. 1972, 14, 305-309.

[89] N. T. Maitra, F. Zhang, R. J. Cave, K. Burke, J. Chem. Phys. 2004, 120, 5932-5937.

[90] L. Goerigk, M. Casanova-Paéz, Aust. J. Chem. 2021, 74, 3-15.

[91] H. B. Schlegel, J. M. Millam, S. S. lyengar, G. A. Voth, A. D. Daniels, G. E. Scuseria, M. J. Frisch, J. Chem. Phys. 2001, 114, 9758-9763.

[92] S. S. lyengar, H. B. Schlegel, J. M. Millam, G. A. Voth, G. E. Scuseria, M. J. Frisch, J. Chem. Phys. 2001, 115, 10291-10302.

[93] A. R. Leach, Molecular Modelling: Principles and Applications, Addison Wesley Longman, Edinburgh, 1996.

[94] C. Hättig, F. Weigend, J. Chem. Phys. 2000, 113, 5154-5161.

[95] TURBOMOLE V6.3 2011, a development of University of Karlsruhe and Forschungszentrum Karlsruhe $\mathrm{GmbH}$, 1989-2007, TURBOMOLE GmbH, since 2007; available from http://www.turbomole.com (accessed July $10,2021)$.

[96] S. G. Balasubramani, G. P. Chen, S. Coriani, M. Diedenhofen, M.S. Frank, Y. J. Franzke, F. Furche, R. Grotjahn, M. E. Harding, C. Hättig, A. Hellweg, B. Helmich-Paris, C. Holzer, U. Huniar, M. Kaupp, A. M. Khah, S. K. Khani, T. Müller, F. Mack, B. D. Nguyen, S. M. Parker, E. Perlt, D. Rappoport, K. Reiter, S. Roy, M. Rückert, G. Schmitz, M. Sierka, E. Tapavicza, D. P. Tew, C. van Wüllen, V. K. Voora, F. Weigend, A. Wodyński, J. M. Yu, J. Chem. Phys. 2020, 152, 184107.

[97] Gaussian 16 (Revision B.01), M. J. Frisch, G. W. Trucks, H. B. Schlegel, G. E. Scuseria, M. A. Robb, J. R. Cheeseman, G. Scalmani, V. Barone G. A. Petersson, H. Nakatsuji, X. Li, M. Caricato, A. V. Marenich, J. Bloino, B. G. Janesko, R. Gomperts, B. Mennucci, H. P. Hratchian, J. V. Ortiz, A. F. Izmaylov, J. L. Sonnenberg, D. Williams-Young, F. Ding, F. Lipparini, F. Egidi, J. Goings, B. Peng, A. Petrone, T. Henderson, D. Ranasinghe, V. G. Zakrzewski, J. Gao, N. Rega, G. Zheng, W. Liang, M. Hada, M. Ehara, K. Toyota, R. Fukuda, J. Hasegawa, M. Ishida, T. Nakajima, Y. Honda, O. Kitao, H. Nakai, T. Vreven, K. Throssell, J. A. Montgomery, Jr., J. E. Peralta, F. Ogliaro, M. J. Bearpark, J. J. Heyd, E. N. Brothers, K. N. Kudin, V. N. Staroverov, T. A. Keith, R. Kobayashi, J. Normand, K. Raghavachari, A. P. Rendell, J. C. Burant, S. S. lyengar, J. Tomasi, M. Cossi, J. M. Millam, M. Klene, C. Adamo, R. Cammi, J. W. Ochterski, R. L. Martin, K. Morokuma, O. Farkas, J. B. Foresman, D. J. Fox, Gaussian, Inc., Wallingford, CT, 2016

[98] N. E. Chayen, M. Cianci, J. G. Grossmann, J. Habash, J. R. Helliwell, G. A. Nneji, J. Raftery, P. J. Rizkallah, P. F. Zagalsky, Acta Crystallogr. Sect. D 2003, 59, 2072-2082.

[99] G. Bartalucci, J. Coppin, S. Fisher, G. Hall, J. R. Helliwell, M. Helliwell, S. Liaaen-Jensen, Acta Crystallogr. Sect. B 2007, 63, 328-337.

[100] E. Walczak, B. Szefczyk, T. Andruniów, J. Chem. Theory Comput. 2013 9, 4915-4927.

[101] J. L. Bao, L. Gagliardi, D. G. Truhlar, J. Phys. Chem. Lett. 2018, 9, 23532358

[102] D. Hait, M. Head-Gordon, J. Phys. Chem. Lett. 2018, 9, 6280-6288.

[103] N. A. Murugan, J. Kongsted, Z. Rinkevicius, H. Ågren, Proc. Natl. Acad. Sci. USA 2010, 107, 16453-16458. 
[104] M. Drobizhev, T. E. Hughes, Y. Stepanenko, P. Wnuk, K. O'Donnell, J. N. Scott, P. R. Callis, A. Mikhaylov, L. Dokken, A. Rebane, Sci. Rep. 2012, 2, 688.

[105] C. Daday, C. Curutchet, A. Sinicropi, B. Mennucci, C. Filippi, J. Chem. Theory Comput. 2015, 11, 4825-4839.

[106] L. Goerigk, J. Moellmann, S. Grimme, Phys. Chem. Chem. Phys. 2009, $11,4611-4620$.

[107] S. Grimme, F. Neese, J. Chem. Phys. 2007, 127, 154116.

[108] D. Mester, M. Kállay, J. Chem. Theory Comput. 2021, 17, 4211-4224.

[109] M. Casanova-Páez, L. Goerigk, J. Chem. Theory Comput. 2021, 17, 5165-5186.
[110] T. Kato, K. Strakova, J. García-Calvo, N. Sakai, S. Matile, Bull. Chem. Soc. Jpn. 2020, 93, 1401-1411.

[111] I. Higuera-Ciapara, L. Félix-Valenzuela, F. M. Goycoolea, Crit. Rev. Food Sci. Nutr. 2006, 46, 185-197.

Manuscript received: August 21, 2021

Revised manuscript received: September 25, 2021

Accepted manuscript online: October 11, 2021

Version of record online: 


\section{ARTICLES}

Fluctuations matter: Comparing static and dynamical approaches to calculating excitation energies for an optically bright state of a prototypical conjugated molecule with distinct potential-energy minima, it is shown that the effects of thermal fluctuations in the conjugation are not negligible, as inherently assumed in the widely used static approach.



Dr. J. Wang, Prof. B. Durbeej*

$1-10$

Thermal Fluctuations in Conjugation and their Effect on Calculated Excitation Energies: A Case Study on the Astaxanthin Carotenoid 